\title{
Existence of Shapiro Steps in the Dissipative Regime in Superconducting Weak Links
}

\author{
Connor D. Shelly $\odot,{ }^{*}$ Patrick See, Ivan Rungger, and Jonathan M. Williams \\ National Physical Laboratory, Hampton Road, Teddington TW11 OLW, United Kingdom
}

(Received 18 October 2019; revised manuscript received 20 January 2020; accepted 24 January 2020; published 26 February 2020)

\begin{abstract}
We present measurements of microwave-induced Shapiro steps in a superconducting nanobridge weak link in the dissipative branch of a hysteretic current-voltage characteristic. We demonstrate that Shapiro steps can be used to infer a reduced critical current and an associated local temperature. Our observation of Shapiro steps in the dissipative branch shows that a finite Josephson coupling exists in the dissipative state. Although the nanobridge is heated, our model shows that the temperature remains below the critical temperature. This work provides evidence that Josephson behavior can still exist in thermally hysteretic weak-link devices and will allow extension of the temperature range over which nanobridge-based singleflux-quantum circuits, micron-sized superconducting quantum interference devices (i.e., nanoSQUIDs), and Josephson voltage standards can be used.
\end{abstract}

DOI: 10.1103/PhysRevApplied.13.024070

\section{INTRODUCTION}

A superconducting weak link (WL) can be realized by creating a narrow constriction between two bulk superconducting electrodes. If the constriction dimensions are made sufficiently small (comparable to $3.5 \xi$, where $\xi$ is the Ginzburg-Landau coherence length), then the WLs are expected to exhibit characteristic Josephson behavior [1]. Nanobridge constrictions can thus be used instead of traditional Josephson tunnel junctions based on oxide barriers, or superconductor-normal-metal-superconductor $(S-N-S)$ junctions. Nanobridge-constriction junctions can be fabricated using only a single-step lithography process and no oxide layer is required. The lack of an oxide barrier also removes a potential source of decoherence, which is an important consideration when building quantum circuits [2]. Nanobridge WLs can be made out of a large range of superconducting materials (both low- and high- $T_{c}$ ), which facilitates incorporation with other circuit elements and nanosensors.

The majority of the work in the area has focused on the development and optimization of micron-sized superconducting quantum interference devices (nanoSQUIDs), which are implemented using two WLs [3]. NanoSQUIDs

\footnotetext{
*connor.shelly@npl.co.uk
}

Published by the American Physical Society under the terms of the Creative Commons Attribution 4.0 International license. Further distribution of this work must maintain attribution to the author(s) and the published article's title, journal citation, and DOI. have application in single-magnetic-nanoparticle detection [4], scanning SQUID microscopy for imaging of nanoscale phenomena [5-7], and nanoelectromechanical-system (NEMS) readout $[8,9]$. Aside from magnetometer-based applications, WL Josephson junctions could be used in place of traditional junctions for single-flux-quantum (SFQ) circuits [10] and Josephson voltage standards for metrology $[11,12]$. Weak links also have utility as Josephson elements in qubits and parametric amplifiers [13-16], as well as for single-quasiparticle trapping and counting [17].

In general, hysteresis is observed in the current-voltage characteristics (IVCs) of WLs. Unlike conventional tunnel junctions, where the hysteresis can be explained by capacitance in the resistively and capacitively shunted junction (RCSJ) model [18], the origin of hysteresis in WL junctions is attributed to heating and subsequent thermal runaway of the junction [19], similar to that observed in $S-N-S$ junctions $[20,21]$. This situation was first described by Skocpol, Beasley, and Tinkham (SBT) [19], who stated that as the bias current $I_{\mathrm{dc}}$ applied to the WL is increased above the critical current $I_{c}$, a "hot-spot" region in the WL forms, in which the local temperature exceeds the critical temperature $T_{c}$. When $I_{\mathrm{dc}}$ is then reduced, the hot spot is maintained by Joule heating. The WL is only able to return to the superconducting state when $I_{\mathrm{dc}}$ is reduced to below the retrapping current $I_{r}$, where $I_{r}<I_{c}$. In recent years, further refinements have been made to the SBT model by inclusion of a temperature-dependent thermal conductivity at temperatures below $T_{c}[22,23]$ and extension of the model to millikelvin temperatures [24]. In addition, recent work has been carried out to understand and reduce the hysteresis in the IVC [25-28]. 
Weak-link thermal models $[19,22,24]$ indicate that with a sufficiently large bias current, the temperature in the WL can exceed $T_{c}$; in some cases, the $T>T_{c}$ region is predicted to extend several micrometers into the electrodes. Indeed, Kumar et al. present a device-state diagram for WL-based nanoSQUIDs showing that at $T<T_{H}$ (where $T_{H}$ is the crossover temperature between the hysteretic and nonhysteretic regimes) and $I_{\mathrm{dc}}>I_{r}$, the WLs and the micron-scale leads are in the resistive state [25]. Preliminary nanoSQUID measurements in the hysteretic regime have shown no magnetic flux dependence of the retrapping current $[23,25]$. However, Biswas et al. have recently demonstrated that thermally optimized nanoSQUIDs do exhibit a dependence of $I_{r}$ on magnetic flux [29], indicating that the Josephson coupling does not completely vanish in the dissipative state. The Josephson effect can also be demonstrated through the observation of microwaveinduced Shapiro steps [30]. We have previously observed Shapiro steps in WLs operated in the nonhysteretic regime [31] and they have also been found in long nanowires in the "phase-slip center" regime [32,33].

In this work, we demonstrate Josephson behavior in hysteretic nanobridge WL junctions by observation of Shapiro steps and combine the experimental data with our model to estimate the local temperature of the WL. To do this, we measure the IVC of the WL at a temperature $T<T_{H}$ while applying a radio-frequency (rf) current. Notably, we observe Shapiro steps on the dissipative branch of the hysteretic IVC, which has previously been thought to be in the fully normal state, where it has been assumed that the nanobridge and parts of the electrode have $T>T_{c}$.

\section{NANOBRIDGE FABRICATION AND MEASUREMENT SETUP}

The WLs are fabricated by electron-beam lithography (EBL) and dry etching. A niobium film of thickness $150 \mathrm{~nm}$ is sputtered onto a silicon substrate, on top of which a 30 -nm-thick aluminum film to be used as a hard mask is defined by EBL and thermally deposited by liftoff. An array of ten nanobridges is defined to a width of $40 \mathrm{~nm}$ and a length of $100 \mathrm{~nm}$. The niobium is then dry etched into the silicon substrate using a $\mathrm{CHF}_{3}-\mathrm{SF}_{6}$ plasma. The aluminum mask is left on.

Electrical measurements of the WLs are carried out in a ${ }^{4} \mathrm{He}$ dip Dewar. The temperature is varied between 4 and $9 \mathrm{~K}$ by varying the position of the probe in the gas column. The IVCs are measured in a four-terminal configuration using an optically isolated measurement unit optimized for high-precision electrical metrology [34]. To observe Shapiro steps, the WL is biased with an rf current. The scaling factor between the applied rf voltage from the synthesizer $V_{\mathrm{rf}}$ and the rf current that reaches the device $I_{\mathrm{rf}}$ is determined from two IVCs (for details,

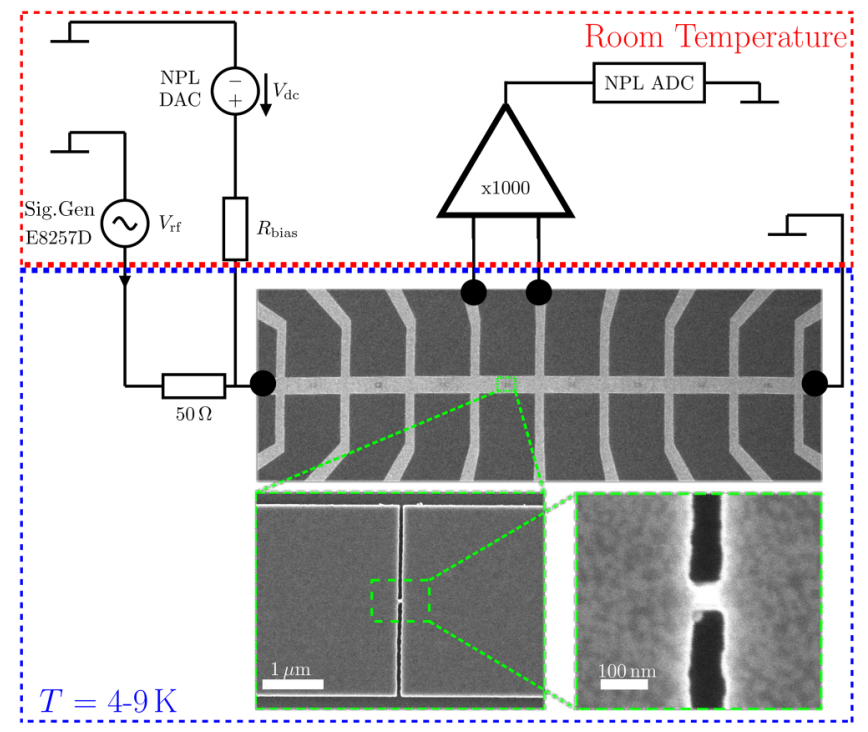

FIG. 1. Scanning electron micrographs of a niobium WL array and measurement schematic. To measure a single WL IVC, a bias current is driven through the entire array via the digital-to-analog converter (NPL-DAC) voltage source and a bias resistor. The voltage across an individual WL is measured using the analogto-digital converter (NPL-ADC). To investigate the influence of rf irradiation, an $\mathrm{rf}$ current is applied to the entire array using an rf synthesizer and on-chip $50 \Omega$ resistor.

see Appendix A). A scanning electron micrograph and measurement schematic are shown in Fig. 1.

\section{EXPERIMENTAL RESULTS}

Typical IVCs measured without an rf current are shown in Fig. 2 at different temperatures. The behavior is qualitatively similar to that observed previously by the authors [31] and elsewhere [22,24,27], showing that both $I_{c}$ and $I_{r}$ are temperature dependent and that the hysteresis disappears above $T_{H}$.

Figure 3(a) shows a differential-resistance map obtained by numerically differentiating IVCs measured with an rf current applied. The color map shows the evolution of the IVC as a function of an increasing applied rf current, where the dark regions of the color map indicate a plateau in the IVC. These plateaus can be seen in the IVC traces and form at the expected Josephson voltages $V=n(h f / 2 e)$, where $f=20 \mathrm{GHz}$ is the frequency of the rf current (the differential conductance is shown in Appendix B, which emphasizes the positions of the constant-voltage Shapirosteps). Figure 3(b) shows three traces from the differentialresistance map. The trace at $I_{\mathrm{rf}}=213 \mu \mathrm{A}$ shows Shapiro steps on the down sweep (the current from a negative $I_{\mathrm{dc}}$ to zero) of the dissipative branch of the hysteretic IVC. The trace at $I_{\mathrm{rf}}=328 \mu \mathrm{A}$ shows that Shapiro steps appear on both the up sweep (the current from zero to positive $I_{\mathrm{dc}}$ ) and the down sweep of the IVC. When sufficiently 

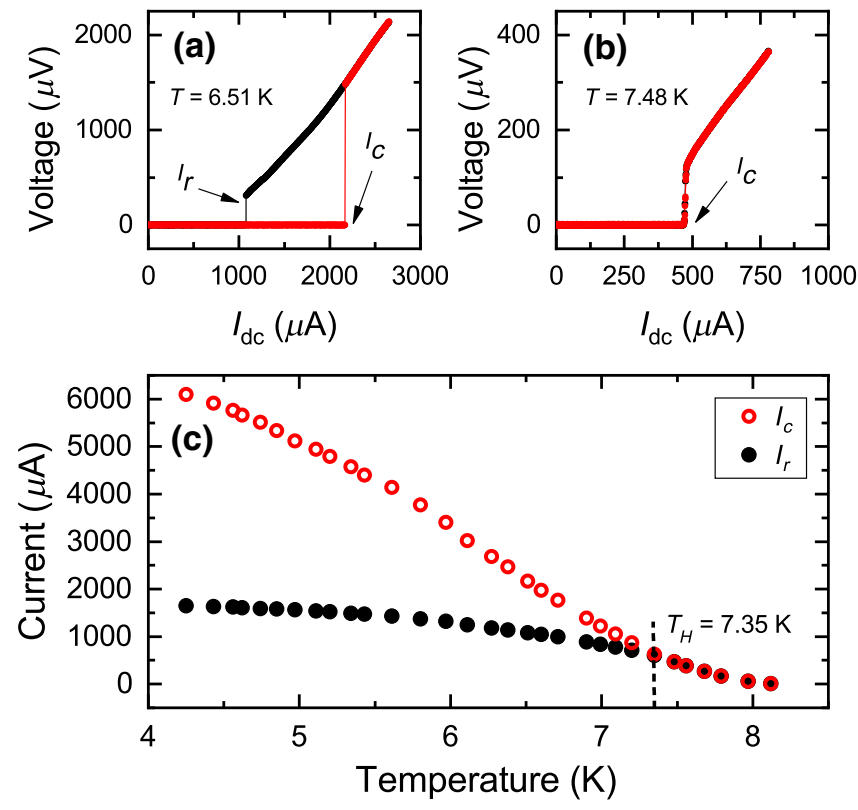

FIG. 2. (a) The IVC of a weak-link nanobridge measured at a bath temperature of $6.51 \mathrm{~K}$, exhibiting thermal hysteresis. (b) The IVC of a weak-link nanobridge at a bath temperature of 7.48 $\mathrm{K}$, showing no hysteresis. (c) The critical current and the retrapping current measured at different bath temperatures. Hysteresis occurs at $T<T_{H}$. For this sample, $T_{H} \approx 7.35 \mathrm{~K}$.

large $\mathrm{rf}$ currents are applied, as shown by the trace taken at $I_{\mathrm{rf}}=407 \mu \mathrm{A}$, the hysteresis in the IVC disappears and the WL behaves as a nonhysteretic junction while still exhibiting Shapiro steps. This behavior is qualitatively similar to that observed by de Cecco et al. in $S-N-S$ Josephson junctions [35]. The existence of Shapiro steps in the hysteretic IVC (at $I_{\text {rf }}<372 \mu \mathrm{A}$ ) on both the up and down sweeps indicates that the WL is not in a fully dissipative state but, instead, provides evidence of a finite Josephson supercurrent existing in the dissipative state, in agreement with the recently observed retrapping current modulation [29]. Similar to de Cecco et al. [35], we observe that at sufficiently high $I_{\mathrm{rf}}(>372 \mu \mathrm{A})$, the IVC become nonhysteretic, likely due to the temperature of the junction at $I_{\mathrm{dc}}=0$ increasing above the crossover temperature $\left(T>T_{H}\right)$.

To explain our observation of Shapiro steps in the dissipative regime, we first consider what happens to the $I_{c}$ of a hysteretic WL. Figure 4 shows IVCs at different rf currents. Both the up and down sweeps are shown. As $I_{\mathrm{dc}}$ is swept from zero to positive values, the junction's initial-state critical current $I_{c}^{0}$ is reached, causing the junction to enter the dissipative regime. Due to Joule heating of the WL in this dissipative regime, the local temperature increases to $T^{*}$. The reduced critical current associated with this temperature is thus described as $I_{c}^{*}=I_{c}\left(T^{*}\right)$. The dissipative region of the IVC is now at this lower critical current. To determine $I_{c}^{*}$, we fit the dissipative region of the measured IVC by numerically solving the first-order differential equation

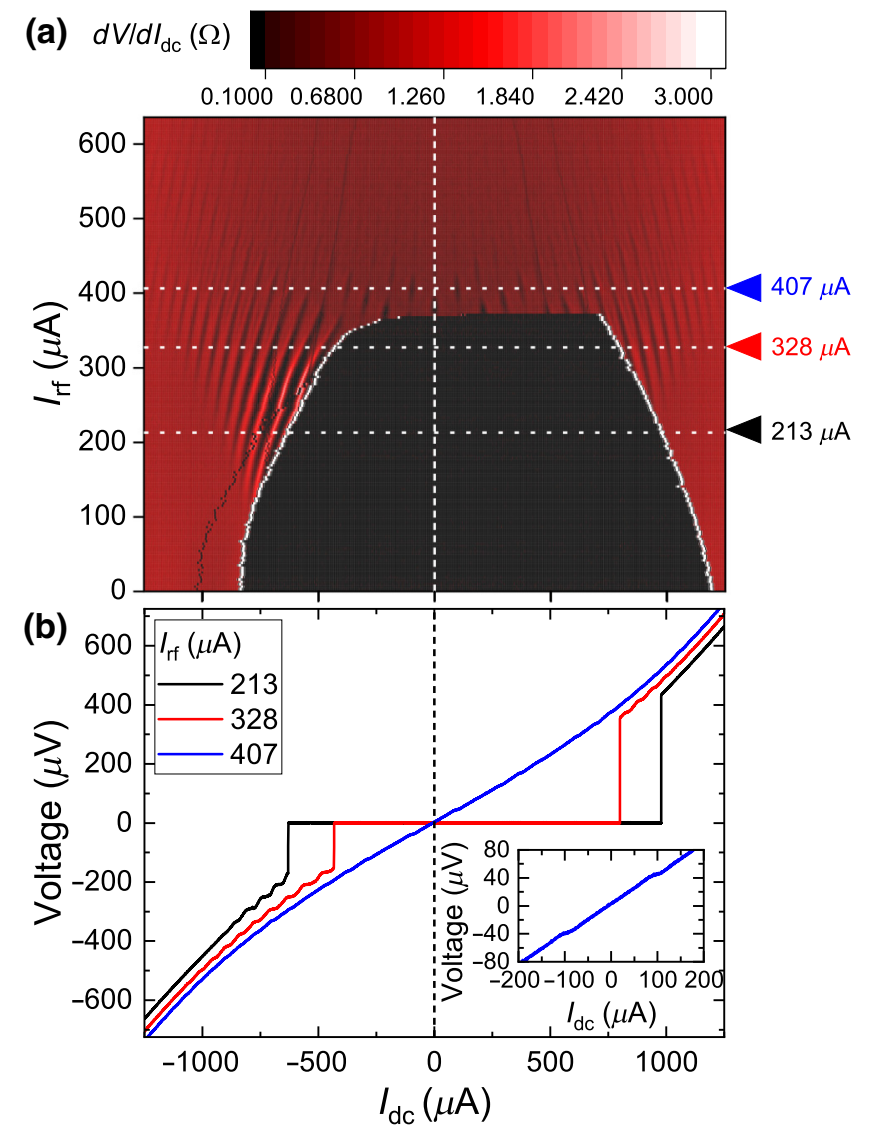

FIG. 3. (a) The differential-resistance color map as a function of $I_{\mathrm{dc}}$ and $I_{\mathrm{rf}}$ measured at a bath temperature of $7 \mathrm{~K}$ (hysteretic regime). The darker regions indicate flat features in the IVC corresponding to Shapiro steps at the expected voltages for $f_{\mathrm{rf}}=20 \mathrm{GHz}$. (b) Selected IVC traces. At $I_{\mathrm{rf}}=213 \mu \mathrm{A}$, steps are observed as $I_{\mathrm{dc}}$ is swept down from negative values to zero. $I_{\mathrm{rf}}=328 \mu \mathrm{A}$ steps are seen on both branches (as the current is swept from negative values to zero and as the current is swept from zero to positive values). When the applied $\mathrm{rf}$ is of sufficient amplitude, the nanobridge no longer shows any evidence of hysteresis in the IVC, as shown by the trace at $I_{\mathrm{rf}}=407 \mu \mathrm{A}$. The inset shows the $I_{\mathrm{rf}}=407 \mu \mathrm{A}$ trace over a smaller $I_{\mathrm{dc}}$ range, showing the first observable step ( $n=1$ step occurring at $V \approx 41.4 \mu \mathrm{V})$.

describing the RSJ model with an applied rf current [36],

$$
\frac{\hbar}{2 e R_{n}} \dot{\phi}+I_{c} \sin (\phi)=I_{\mathrm{dc}}+I_{\mathrm{rf}} \sin (2 \pi f t)
$$

where we assume a sinusoidal current-phase relation. We use the trace with $I_{\mathrm{rf}}=0$ in order to determine a value for the normal-state resistance $R_{n}$, which we keep constant for all other IVC fits. The fit to the IVC at $I_{\mathrm{rf}}=0$ is shown in Figure 4(a), with fitting parameters of $I_{c}^{*}=690 \mu \mathrm{A}$ and $R_{n}=0.55 \Omega$. Figures $4(\mathrm{~b})-4(\mathrm{e})$ show RSJ model fits to the measured IVC at different $I_{\mathrm{rf}}$ values, using $I_{c}^{*}$ as the only fitting parameter. As $I_{\mathrm{rf}}$ is increased, the additional 


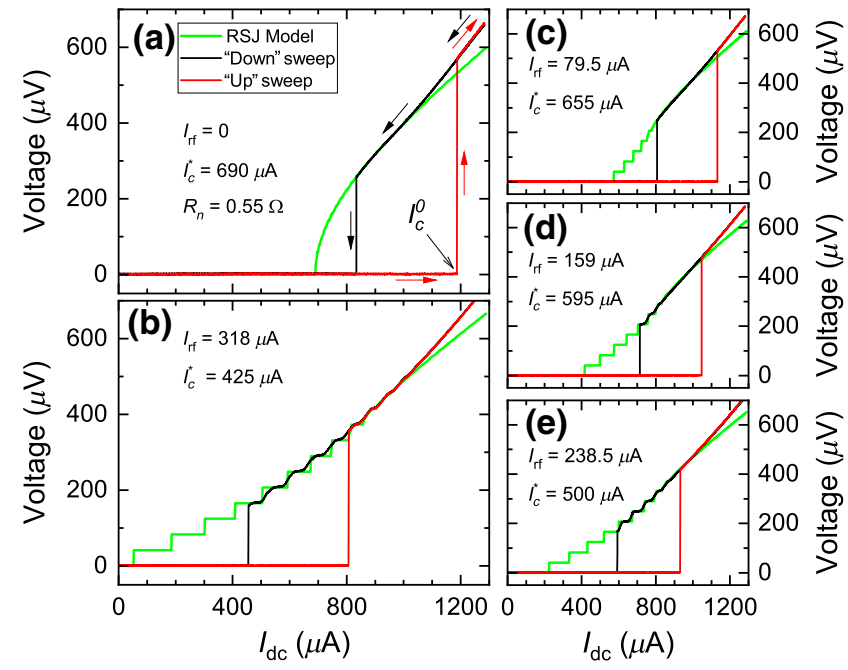

FIG. 4. IVC traces shown at different $V_{\text {rf }}$ amplitudes: the "up" sweep shown in red, the "down" sweep shown in black, and the RSJ model shown in green. (a) The IVC at $I_{\text {rf }}=0$ used to find $I_{c}^{*}$ and $R_{n}$. The "up" sweep shows that as the initial-state critical current $I_{c}^{0}$ is reached, the junction transitions to a lower critical current $I_{c}^{*}$. During the "down" sweep, the junction remains on this path until the Joule heating is no longer sufficient to stop the junction re-entering the fully superconducting regime. The RSJ model is used to determine $I_{c}^{*}$ and $R_{n} . R_{n}$ is kept constant for the remainder of the analysis. (b) The IVC with an applied $\mathrm{rf}$ of $I_{\mathrm{rf}}=$ $318 \mu \mathrm{A}$. Our model uses $I_{c}^{*}$ as the only fitting parameter and is able to reproduce the Shapiro-step position and the total number of steps for the full range of applied $I_{\mathrm{rf}}$ in the hysteretic region of the color map shown in Fig. 3. Measured IVC and RSJ model fits at (c) $I_{\mathrm{rf}}=79.5 \mu \mathrm{A}$, (d) $I_{\mathrm{rf}}=159 \mu \mathrm{A}$, and (e) $I_{\mathrm{rf}}=238.5 \mu \mathrm{A}$.

dissipated power leads to an increase in $T^{*}$ and a corresponding reduction of $I_{c}^{*}$. The RSJ model with $I_{c} \rightarrow I_{c}^{*}$ reproduces both the position of the Shapiro steps and the number of observable steps. At higher $I_{\mathrm{dc}}$, the data and model deviate, which we attribute to a further increase in temperature due to Joule heating and subsequent modification of $I_{c}^{*}$ and $R_{n}$. Our fitting also predicts the existence of steps below $I_{r}$, which are inaccessible in our measurement. This is attributed to the reduction in Joule heating as $I_{\mathrm{dc}}$ is reduced, which leads to a decrease of the local temperature and thus an increase of $I_{c}^{*}$ until it is in excess of $I_{\mathrm{dc}}$, at which point the WL enters the fully superconducting state again and the local temperature returns approximately to the bath temperature, $T_{\text {bath }}$.

The best-fit values of $I_{c}^{*}$ found at each $I_{\mathrm{rf}}$ are shown in Fig. 5(a). As $I_{\mathrm{rf}}$ is increased, the value of $I_{c}^{*}$ reduces, as discussed above. The vertical dashed line in the figure denotes the crossover from hysteretic to nonhysteretic junction behavior. After this point, the steps in the IVC have less contrast and fitting is done using numerically differentiated data. Only one point in this region is fitted, to demonstrate that there is no large discontinuity beyond the crossover line. An estimate of the local temperature of the WL is
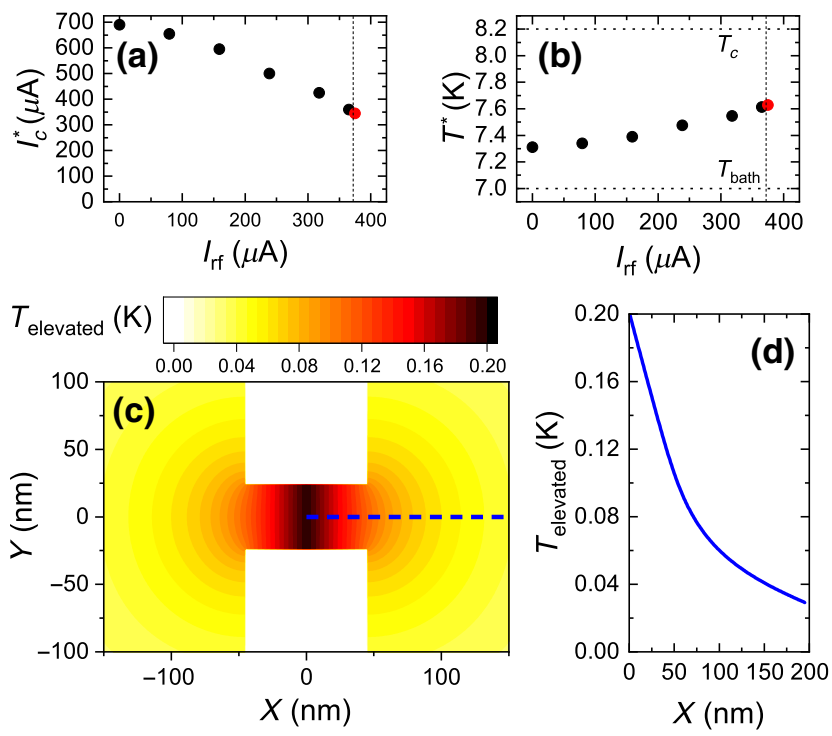

FIG. 5. (a) The best fit of $I_{c}^{*}$ at different $I_{\mathrm{rf}}$. As $I_{\mathrm{rf}}$ is increased, $I_{c}^{*}$ reduces. (b) The temperature inferred from the value of $I_{c}^{*}$ and the $I_{c}(T)$ shown in Fig. 2(c). The dashed vertical lines in (a) and (b) indicate the $I_{\mathrm{rf}}$ value beyond which the IVCs are nonhysteretic (i.e., $I_{\mathrm{rf}}>372 \mu \mathrm{A}$ in Fig. 3). The fits to the RSJ model beyond this line (shown in red) are harder to achieve due to reduced step contrast. (c) The modeled temperature elevation in the nanobridge and electrodes. The power dissipation calculated from Shapiro step 6 of the IVC data in Fig. 4(b). The temperature elevation at the center of the nanobridge is approximately 0.2 $\mathrm{K}$. (d) The elevated temperature along the nanobridge, extending into the electrodes [data taken along the blue dotted line in (c)].

made using the $I_{c}(T)$ data from Fig. 2(c) and the results are shown in Fig. 5(b). Our fitting procedure gives an estimate of $I_{c}^{*}$ and thus the WL local temperature for values of $I_{\mathrm{dc}}$ in the vicinity of the hysteresis loop.

\section{DISCUSSION AND THERMAL MODELING}

In a WL operated in the dissipative regime, the temperature is not constant but is expected to be highest in the center of the bridge and to decrease within the electrodes. The estimated $T^{*}$ is therefore the local WL temperature and corresponds to the equivalent bath temperature of a WL with critical current $I_{c}^{*}$ at $I_{\mathrm{rf}}=0$ [see Fig. 2(c)]. We are interested in this local temperature at the center of the bridge in order to determine whether the bridge is heated into a normal-state $(S-N-S)$ junction or remains in a superconducting state $\left(S-S^{\prime}-S\right)$, where $\mathrm{S}^{\prime}$ represents a superconducting region with modified properties. To determine the temperature profile of the weak link, we employ a finite-element numerical method (for additional details, see Appendix C) in the immediate proximity of the bridge, in combination with an analytical solution in the electrodes, which has a radial temperature distribution. The radial temperature distribution is modeled with a modified Bessel function of the second kind $\left(K_{n}(\gamma r)\right)$ and tends to $T_{\text {bath }}$ at 
large distances [19,22]:

$$
T(r)=\theta K_{0}(\gamma r)+T_{\text {bath }},
$$

where $\theta$ is a constant to be determined. The constant $\gamma=$ $\sqrt{\alpha / \kappa h}$ is the thermal healing length, where $\kappa$ is the thermal conductivity, $\alpha$ is the substrate heat-loss coefficient, and $h$ is the film thickness. The input electrical power $P_{0}$ to the model is taken from a Shapiro-step position on an IVC and is then assumed to be dissipated in a narrow strip at the center of the bridge $(X=0)$. At the interface between the numerical and analytical solutions, the same power is assumed to flow across the interface (which means that no power is lost to the substrate up to this interface; thus we consider $\alpha=0$ in this region). The power at the interface is given by

$$
P_{0}=-\left.\kappa \pi r_{1} h \frac{d T_{1}}{d r}\right|_{r=r_{1}},
$$

where $r_{1}$ is the boundary radius. The temperature gradient at $r_{1}$ is given by

$$
\left.\frac{d T_{1}}{d r}\right|_{r=r_{1}}=-\theta \gamma K_{1}\left(\gamma r_{1}\right)
$$

From these relations, the constant $\theta$ representing the interface temperature can be determined:

$$
\theta=\frac{P_{0}}{\pi \kappa h \gamma r_{1} K_{1}\left(\gamma r_{1}\right)} .
$$

Beyond the boundary radius $r_{1}$, the heat loss to the substrate is taken as $\alpha=10000 \mathrm{~W} \mathrm{~m}^{-2} \mathrm{~K}^{-1}$ (see, for instance, Hazra et al. [22]). The thermal conductivity estimated from the IVC with no rf power dissipated in the bridge [Fig. 4(a)] is found to be $\kappa=4.1 \mathrm{~W} \mathrm{~m}^{-1} \mathrm{~K}^{-1}$.

The thermal conductivity inferred from the electrical $R(T)$ measurements of a superconducting nanobridge, as discussed in Appendix D, is found to be $\kappa=$ $5.2 \mathrm{~W} \mathrm{~m}^{-1} \mathrm{~K}^{-1}$, which agrees to within $22 \%$ of the value determined from the numerical thermal model discussed above.

For the thermal modeling of the nanobridge inclusive of the rf power, we have used the experimentally determined thermal conductivity of $\kappa=5.2 \mathrm{~W} \mathrm{~m}^{-1} \mathrm{~K}^{-1}$. Figure 5(c) shows the results of the numerical modeling for the nanobridge at $T_{\text {bath }}=7 \mathrm{~K}$. The power dissipation input to the model is found from the experimental IVC data of the sixth Shapiro step in Fig. 4(b), $P=150 \mathrm{nW}$. The temperature elevation above $T_{\text {bath }}$ is found to be approximately $0.2 \mathrm{~K}$ [see Figs. 5(c) and 5(d)]. This temperature elevation is lower than the inferred $T^{*}$ shown in Fig. 5(b), but this is accounted for by including power dissipation in the onchip $50 \Omega$ resistor due to the rf current, which is discussed in Appendix E.
From both the inferred $T^{*}$ from our Shapiro-step data and the thermal modeling of our nanobridge, we demonstrate that the dissipative branch of the IVC (in which Shapiro steps are observed) is an $S-S^{\prime}-S$ junction with a reduced critical current $I_{c}^{*}$ and not an $S-N-S$ junction. This result is contrary to previous work in which a normal region has been inferred to extend many microns into the electrodes, with no Josephson behavior observed on the retrapping branch [23-25,27].

\section{CONCLUSION}

In conclusion, we present experimental evidence of a finite Josephson supercurrent existing in the dissipative state of WL Josephson junctions, demonstrated by the existence of Shapiro steps on the retrapping branch of the IVC. We use the RSJ model in combination with the reduced critical current $I_{c}^{*}$ to describe the evolution of the Shapiro steps over the full range of our hysteretic data and to infer a local temperature of the WL. From the existence of Shapiro steps, a nonvanishing Josephson supercurrent, and our thermal modeling, we show that our bridge temperature does not exceed $T_{c}$.

The existence of a Josephson supercurrent demonstrates that WLs may be operated as Josephson junctions even in the dissipative state. This has relevance to the operation of hysteretic WL-based nanoSQUIDs, as well as demonstrating that $\mathrm{rf}$ irradiation can be used as a probe of Josephson behavior in the dissipative regime of single WLs. It is critical to the understanding of WLs for use in applications such as SFQ circuits and Josephson voltage standards, where responses to gigahertz pulses are important. The Shapiro steps can be used as a tool with which to investigate WL behavior as well as to inform the optimization of WL junctions and SQUIDs. Different geometries, materials, and thermal shunts can be investigated using this $\mathrm{rf}$ irradiation technique.

\section{ACKNOWLEDGMENTS}

This work is funded as part of a feasibility study funded by Innovate UK (Project No. 102677). Cofunding is supported by the United Kingdom National Measurement System (NMS) Electromagnetics and Time Programme and the Engineering and Physical Sciences Research Council (EPSRC). We thank P. J. Meeson, A. Ya. Tzalenchuk, V. T. Petrashov, J. Burnett, T. Lindström, and J. Ireland for useful technical discussions and J. C. Gallop and S. E. de Graaf for critical reading of the manuscript. We also thank J. P. Griffiths and G. A. C. Jones for the electron-beam lithography.

\section{APPENDIX A: SCALING OF $I_{\mathrm{rf}}$}

It is necessary to ascertain the scaling between the applied $V_{\text {rf }}$ from the room-temperature rf synthesizer and 


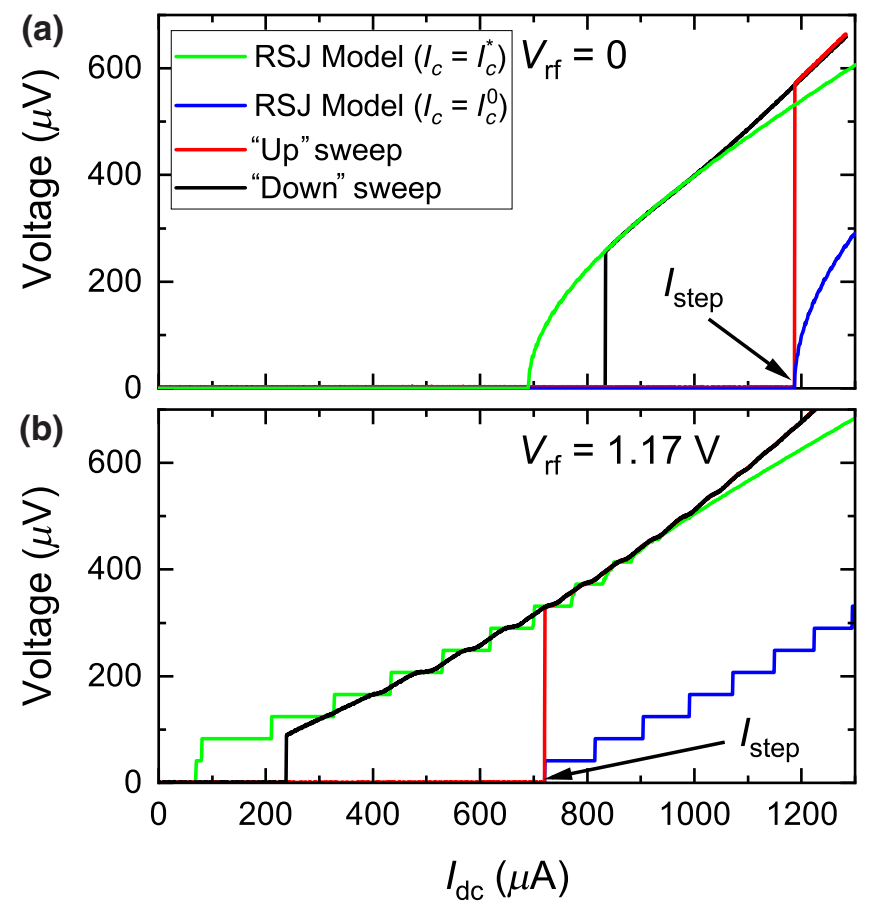

FIG. 6. The measured IVC for two values of $V_{\mathrm{rf}}$ (a) $V_{\mathrm{rf}}=0$ and (b) $V_{\text {rf }}=1.17 \mathrm{~V}$ - shown in red (up sweep) and black (down sweep). Solutions of the RSJ model are shown with $I_{c}=I_{c}^{0}$ (blue) and $I_{c}=I_{c}^{*}$ (green). The position of the transition to the dissipative state is shown and denoted as $I_{\text {step. After } I_{\text {step }} \text { is }}$ reached, the remainder of the blue curve is inaccessible, as due to Joule heating the junction enters a state with a suppressed critical current $I_{c}^{*}$.

the rf current that reaches the weak-link (WL) array $I_{\mathrm{rf}}$. From the measured current-voltage characteristic (IVC), we can determine, for a given $V_{\mathrm{rf}}$, the dc current $I_{\text {step }}$ at which the voltage across the WL becomes nonzero (using the initial-state critical current $I_{c}^{0}$ ). We then use the RSJ model, with $I_{\text {step }}$ as an input parameter, to determine the corresponding rf current $I_{\mathrm{rf}}$ for two cases. This is shown in Fig. 6.

An interpolation between these two fit points using the RSJ model $I_{\text {step }}$ values gives a scaling between $V_{\text {rf }}$ and $I_{\mathrm{rf}}(1 \mathrm{~V}=318 \mu \mathrm{A})$, which gives excellent agreement between the step width and the evolution over the full range of the hysteretic data, as shown in Fig. 4 of the main text.

\section{APPENDIX B: STEPS IN DIFFERENTIAL-CONDUCTANCE COLOR MAP}

For completeness, the IVCs used to produce the differential-resistance color map of the main text are used here to produce a differential-conductance color map, as shown in Fig. 7. This shows clearly that the Shapiro steps are at the expected positions $V=n(h f / 2 e)$ for the applied frequency of $20 \mathrm{GHz}$.
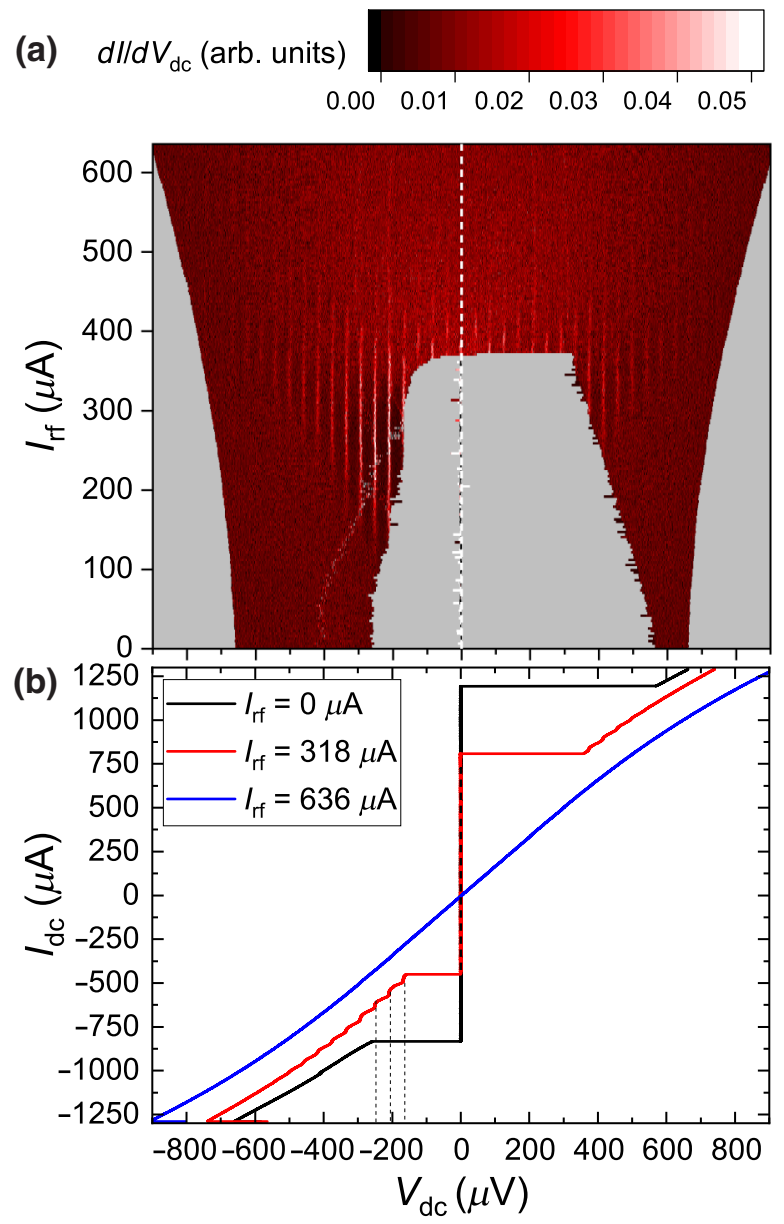

FIG. 7. (a) The $d I / d V_{\mathrm{dc}}$ color map, showing straight Shapiro plateaus at the expected voltages $V=n(h f / 2 e)$. (b) $V_{\mathrm{dc}} \mathrm{vs} I_{\mathrm{dc}}$ for three different $r f$ current values. The dotted lines denote the fourth, fifth, and sixth Shapiro steps.

\section{APPENDIX C: FINITE-ELEMENT NUMERICAL MODELING}

The modeling described in Sec. IV is implemented as a finite-element numerical method and the nanobridge is represented using a grid of square cells as shown in Fig. 8 . Due to symmetry, only one quarter of the nanobridge is required for the simulation.

The sensitivity of the numerical model to the cell size is tested over the range from $1 \mathrm{~nm}$ to $5 \mathrm{~nm}$ and is found to have an effect of less than $10 \mathrm{mK}$. The boundary radius $r_{1}$ is tested over the range from $50 \mathrm{~nm}$ to $150 \mathrm{~nm}$ and is found to have an effect of less than $30 \mathrm{mK}$. The model is linear and thus the calculated temperature profile scales linearly with both the input power and the inverse of the thermal conductivity, $\kappa$. The heat loss to the substrate beyond $r_{1}$ is taken as $\alpha=10000 \mathrm{~W} \mathrm{~m}^{-2} \mathrm{~K}^{-1}$.

The thermal conductivity from the center of the nanobridge to the bath is estimated from the IVC when no rf power is dissipated [see Fig. 4(a)]. The power dissipated 


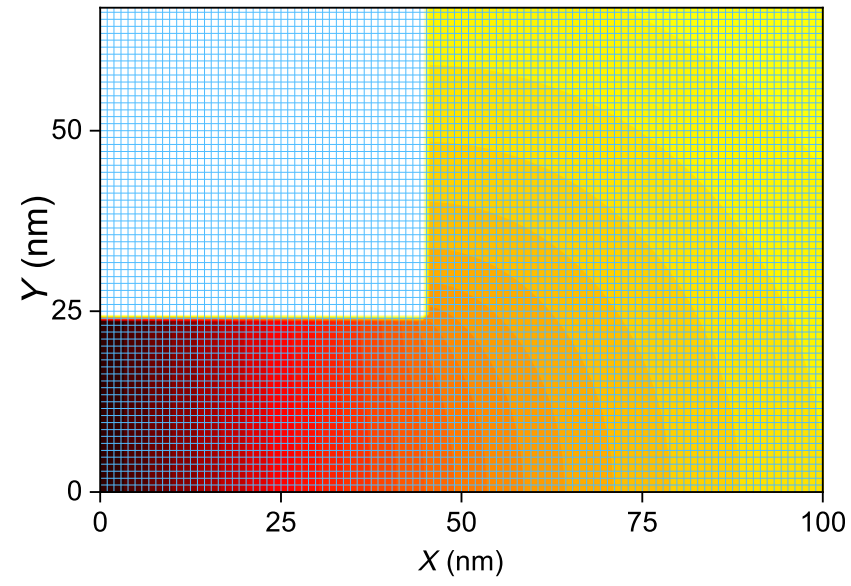

FIG. 8. The finite-element numerical modeling of the nanobridge WL [the color map shows one quadrant of the nanobridge temperature map shown in Fig. 5(c)]. Due to the symmetry of the device, only one quarter of the device requires simulation. The device dimensions are input to the model and constructed using a grid of square cells. The cell size used in the main text is $1 \mathrm{~nm}$, which is the size of the cells shown in the image above. The $r_{1}$ used in this example plot is $65 \mathrm{~nm}$.

in the bridge at $I_{\mathrm{dc}}=800 \mu \mathrm{A}$ is $192 \mathrm{nW}$. The inferred temperature rise $\left(T^{*}-T_{\text {bath }}\right)$ is $0.31 \mathrm{~K}$, resulting in a thermal conductance of $\lambda=619 \mathrm{nW} \mathrm{K}^{-1}$. For our device dimensions, the thermal model gives a thermal conductivity of $\kappa=4.1 \mathrm{~W} \mathrm{~m}^{-1} \mathrm{~K}^{-1}$. This is within $22 \%$ of the thermal conductivity found from electrical $R(T)$ measurements.

\section{APPENDIX D: $R(T)$ MEASUREMENTS AND DETERMINATION OF $\kappa$}

To determine the $T_{\mathrm{c}}$ of our WL, we measure $R(T)$ using the ac lock-in technique suggested in Ref. [37]. We observe a double transition in the data (see Fig. 9) and use the (a)

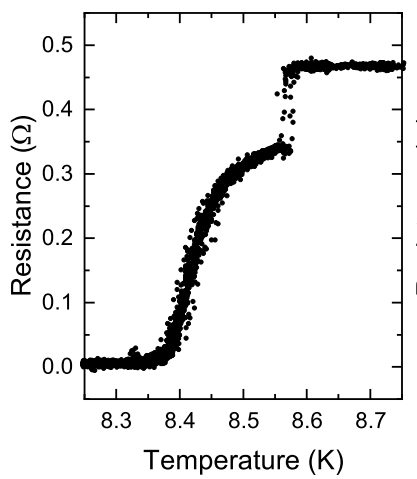

(b)

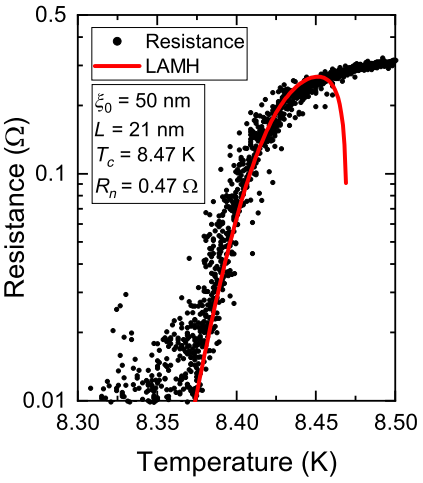

FIG. 9. (a) The resistance as a function of the bath temperature $R(T)$, for a weak-link nanobridge. (b) $R(T)$ plotted over a small temperature range. The solid blue curve is the fit to the LAMHTAPS model. The fit parameters are shown on the figure.
Langer-Ambegaokar-McCumber-Halperin thermally activated phase-slip (LAMH-TAPS) model to determine $T_{c}$ [38]. The good agreement with the data suggests that the double transition is due to thermally activated phase slips. Most of the measurements in the main text are performed at a bath temperature away from this region.

From the $R(T)$ data, we determine the resistivity of our film to be $\rho=33 \mathrm{n} \Omega \mathrm{m}$ (using $R=0.35 \Omega, h=150 \mathrm{~nm}$, $w=50 \mathrm{~nm}$, and $l=80 \mathrm{~nm}$ ). Using the Wiedemann-Franz law to relate the electronic conductivity $\rho$ to the thermal conductivity $\kappa$, we find $\kappa=5.2 \mathrm{~W} \mathrm{~m}^{-1} \mathrm{~K}^{-1}$ [39].

\section{APPENDIX E: EXCESS HEATING FROM $50 \Omega$ LOAD}

We note that in the main text there is a small discrepancy between the elevated temperature found using the thermal model and the elevated local temperature $T^{*}$ inferred from the Shapiro-step data. Figure 5(d) in the main text gives $T_{\text {elevated }}=0.2 \mathrm{~K}$ while $T^{*}=7.55 \mathrm{~K}$, which is thus $0.55 \mathrm{~K}$ above $T_{\text {bath }}$ (but still well below $T_{c}$ ). To account for this discrepancy, we consider the effects of power dissipation in the $50 \Omega$ on-chip resistor due to the rf current.

The calculated thermal conductivity as shown in previous section can then be used to determine the temperature rise due only to dc power for each rf current IVC presented in Fig. 4 of the main text:

$$
\Delta T_{\mathrm{dc}}=\frac{P_{\mathrm{dc}}}{\lambda} .
$$

Therefore, the excess temperature increase due to the power dissipated in the $50 \Omega$ resistor can be calculated:

$$
\Delta T_{\mathrm{rf}}=\left(T^{*}-T_{\mathrm{bath}}\right)-\Delta T_{\mathrm{dc}} .
$$

A graph showing $\Delta T_{\mathrm{rf}}$ plotted vs $P_{\mathrm{rf}}$ (i.e., $I_{\mathrm{rf}}^{2} R$ ) is shown in Fig. 10. The linear dependence between $\Delta T_{\mathrm{rf}}$ and $P_{\mathrm{rf}}$ suggests that the rf heating is proportional to the $\mathrm{rf}$ power.

\section{APPENDIX F: "FLATNESS" OF STEPS}

Figure 11 is the measured differential resistance $d V / d I_{\mathrm{dc}}$ of a representative nanobridge. From Fig. 11, it is possible to see that the lower-order Shapiro steps reach a zero resistance value and then remain at zero resistance (within the resolution of the experiment) over a range of $\mathrm{dc}$ current (approximately $100 \mu \mathrm{A}$ for the first observed step).

\section{APPENDIX G: CORRECTION OF BIAS CURRENT DUE TO SWITCHING OF NEIGHBORING WEAK LINKS}

The individual nanobridge weak links are measured in a typical four-terminal configuration, as shown in Fig. 1 in the main text. However, as our WLs are fabricated in arrays 


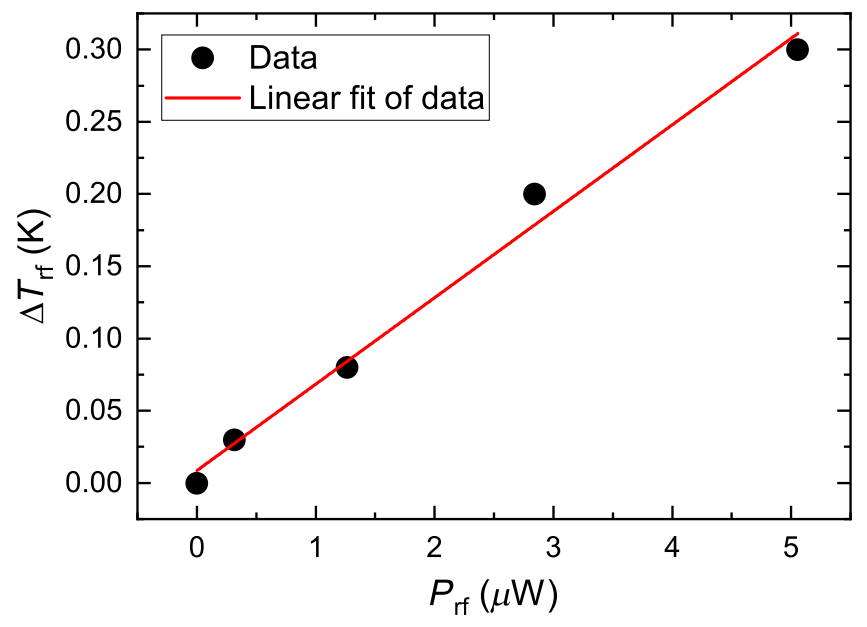

FIG. 10. A plot of $\Delta T_{\mathrm{rf}}$ vs $P_{\mathrm{rf}}$ showing linear dependence.

of ten WLs, we probe only one at a time but drive both $\mathrm{dc}$ and $\mathrm{rf}$ current through the entire array. As a result of this, the true dc bias value applied to the device may have a small abrupt change associated with a switch of a neighboring WL. This can be seen as the feature in the color map in Fig. 3 of the main text, arising close to $-1000 \mu \mathrm{A}$. For the purpose of fitting to the RSJ model in Fig. 4 of the main text, the traces that contain the small change in true bias current are shifted to account for the switching neighboring WL. A before-and-after example of the shifted curves is shown in Fig. 12 for an IVC taken at $I_{\mathrm{rf}}=270 \mu \mathrm{A}$.

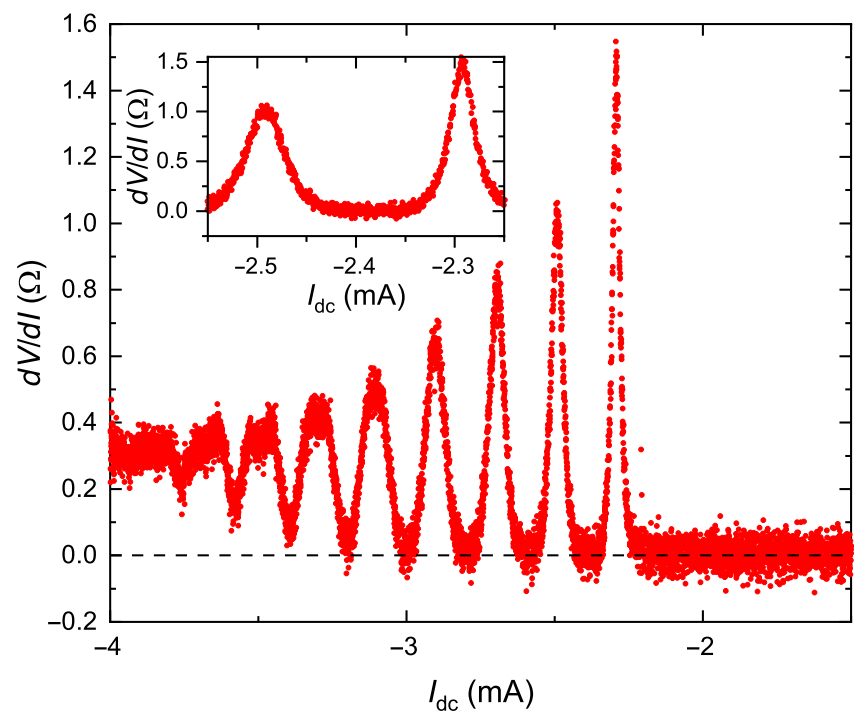

FIG. 11. $d V / d I_{\mathrm{dc}}$ of a representative nanobridge, showing lower-order steps reaching a zero resistance state and persisting in this state over a range of dc bias current. The inset shows the first observable Shapiro step - it is flat (within the resolution of the experiment) over approximately $100 \mu \mathrm{A}$.

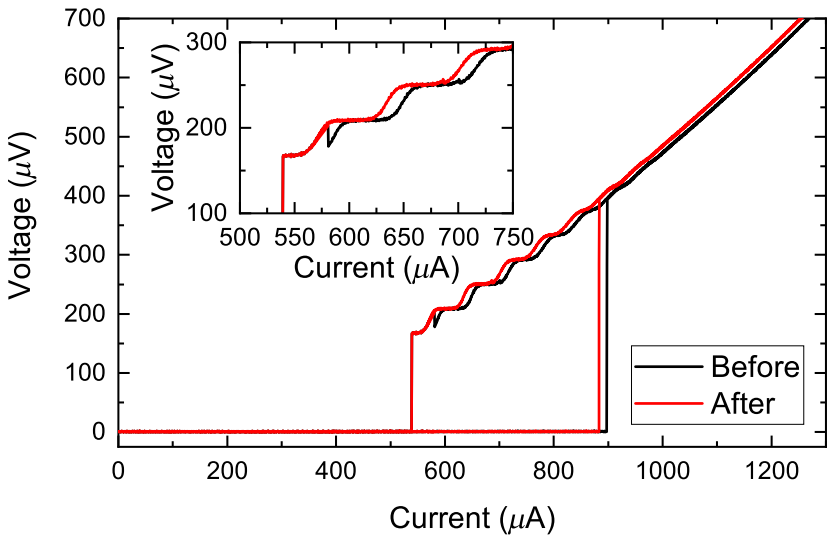

FIG. 12. The measured IVC at $I_{\mathrm{rf}}=270 \mu \mathrm{A}$, showing the discontinuity in the measured data due to switching of a neighboring WL. The inset shows an enlarged plot of the measured data before and after, accounting for a neighboring junction shift. The shift is $15 \mu \mathrm{A}$, less than $0.05 \%$ of the total bias current range.

\section{APPENDIX H: NUMBERING OF INTEGER SHAPIRO STEPS AND HINTS OF SUBHARMONIC SHAPIRO STEPS}

Figure 13 shows the labeling of the integer Shapiro steps in our region of interest. We also highlight hints of subharmonic steps at $I_{\mathrm{rf}}>318 \mu \mathrm{A}$. They are observed as dips in $d V / d I$ but the corresponding inflections are not observable in the IVC. These subharmonic steps might be explained either by including a higher-order harmonic in the supercurrent term of the RSJ model or, alternatively, by effects of external electrical loading of the junction $[40,41]$.

\section{APPENDIX I: THE RSJ MODEL AND A NONSINUSOIDAL $I_{s}(\phi)$ RELATION}

In general, the supercurrent $I_{\mathrm{s}}$ in a Josephson junction as a function of the phase difference $\phi$ has a tilted sinusoidal shape. For lengths $L<3.5 \xi$ it is single-valued and for longer lengths it becomes multivalued [42] and is no longer originated by a coherent interaction of the phases on the two sides of the weak link. The equations for the Dayem bridge Josephson junction supercurrent-phase relationship are given in the literature [43] and can generally be written as $I_{s}=I_{c} f(\phi, L)$, where $f(\phi, L)$ is an oscillating function in $\phi$ with a period $2 \pi$ and an amplitude equal to one. The detailed shape of $f(\phi, \mathrm{L})$ depends on the length of the Dayem bridge. In Fig. 14, we plot $f(\phi, L)$ calculated using the equations given in Ref. [43], for $L=\xi$ and $L=3 \xi$, beyond which the curve becomes multivalued. In general, for small $L, f(\phi, L) \approx \sin (\phi)$; while for increasing $L$, the curves progressively tilt away from the ideal sine relation.

We use the values of $I_{c}$ from Fig. 4 in the main text, which are fitted assuming a perfectly sinusoidal CPR, and evaluate the IVC using $f(\phi, L=3 \xi)$ and compare it to the results obtained using $I_{s}=I_{c} \sin (\phi)$. As shown in Figs. 


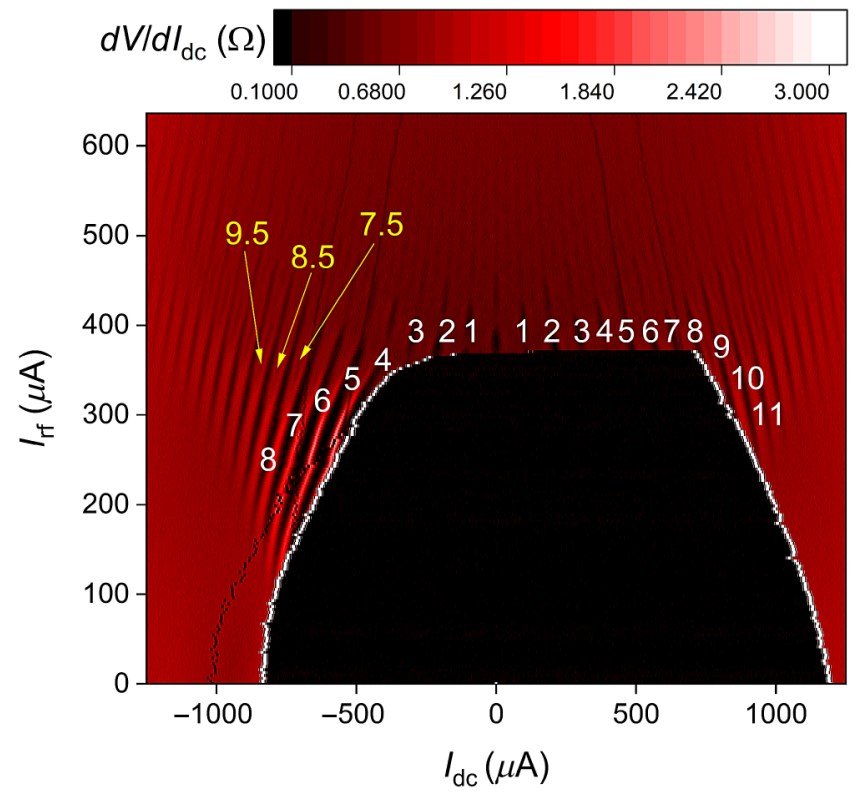

FIG. 13. A differential-resistance color map [Fig. 3(a) from the main text] shown with Shapiro-step numbers demonstrating integer Shapiro steps and hints of subharmonic steps at $I_{\mathrm{rf}}>318 \mu \mathrm{A}$.

15(a)-15(e), the IVCs at all values of $I_{\mathrm{rf}}$ are essentially identical for both cases. This shows that our RSJ model fit to the experimental data does not depend significantly on the detailed shape of the $f(\phi, L)$ relation.

\section{APPENDIX J: TEMPERATURE DEPENDENCE OF DIFFERENTIAL-RESISTANCE MAPS}

We observe Shapiro steps in the dissipative branch of a number of nanobridge devices. Here, we show the

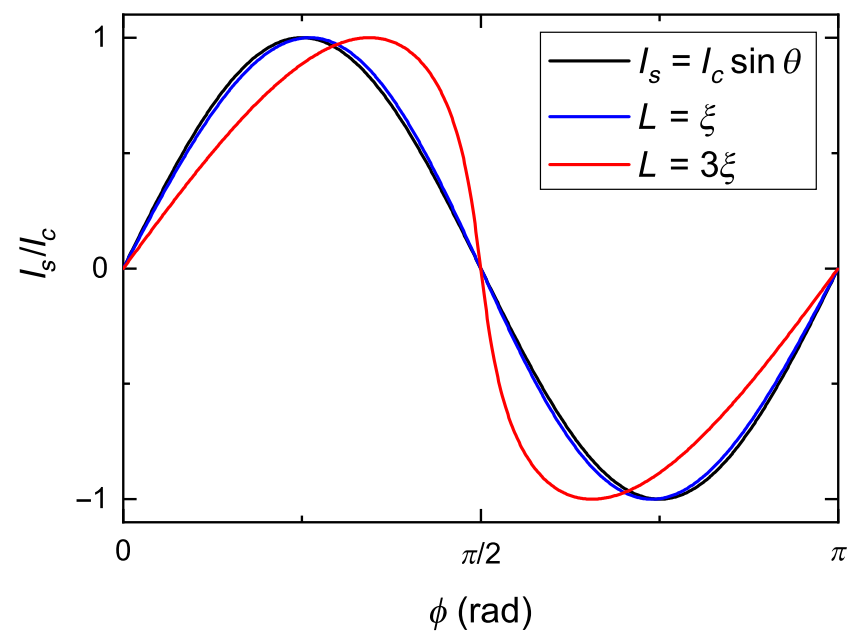

FIG. 14. The Dayem bridge Josephson junction supercurrentphase relationship for different lengths, $L$, of the bridge. The ideal sinusoidal relation is plotted for comparison.
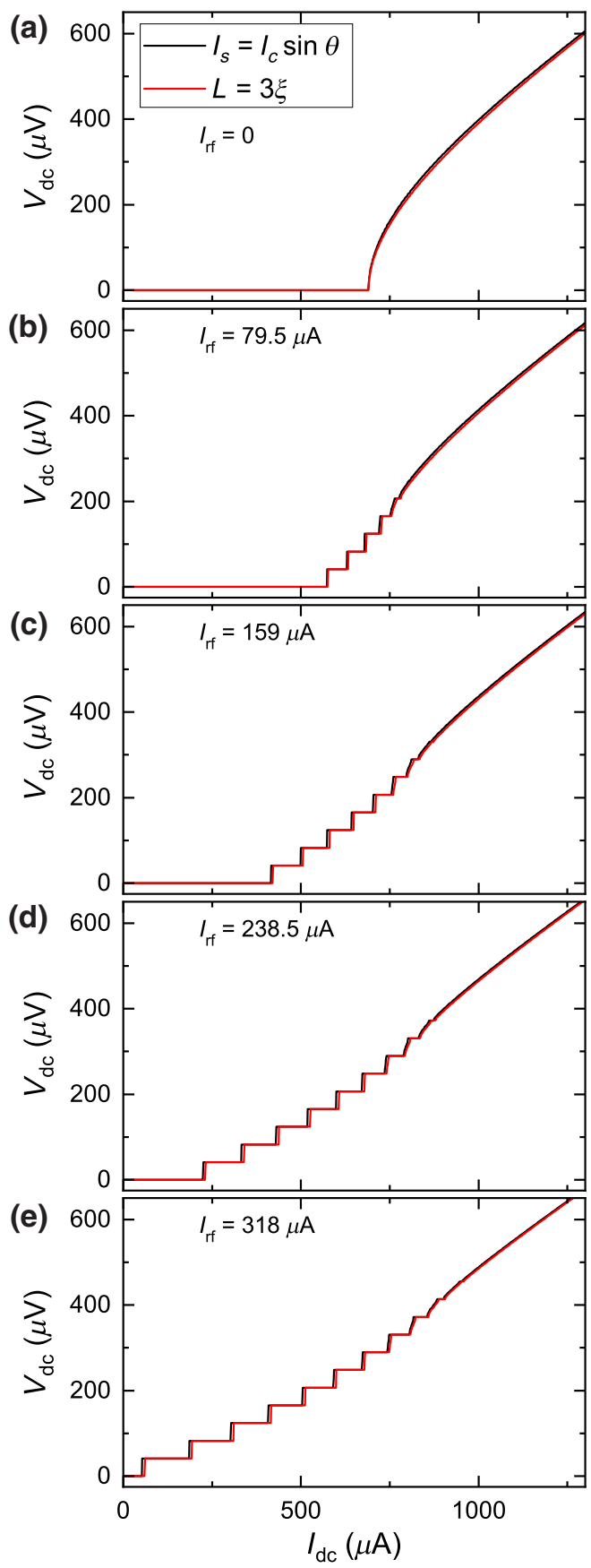

FIG. 15. RSJ model results for $V_{\mathrm{dc}}$ as a function of $I_{\mathrm{dc}}$ for different values of $I_{\mathrm{rf}}$. The black curve uses the sinusoidal supercurrent-phase relationship $I_{s}=I_{c} \sin (\phi)$ as in Fig. 4 of the main text, while for the red curve we use $I_{s}=I_{c} f(\phi, L=3 \xi)$. The curves are almost identical for both cases, showing that the fitting is not sensitive to a change in the supercurrent-phase relationship. The resulting IVCs exhibit the same step widths and number of steps.

differential-resistance color maps of a nanobridge device with a lower critical current. The differential-resistance color maps are taken at different temperatures and show the reduction in $I_{c}$ as the temperature is increased. It is 

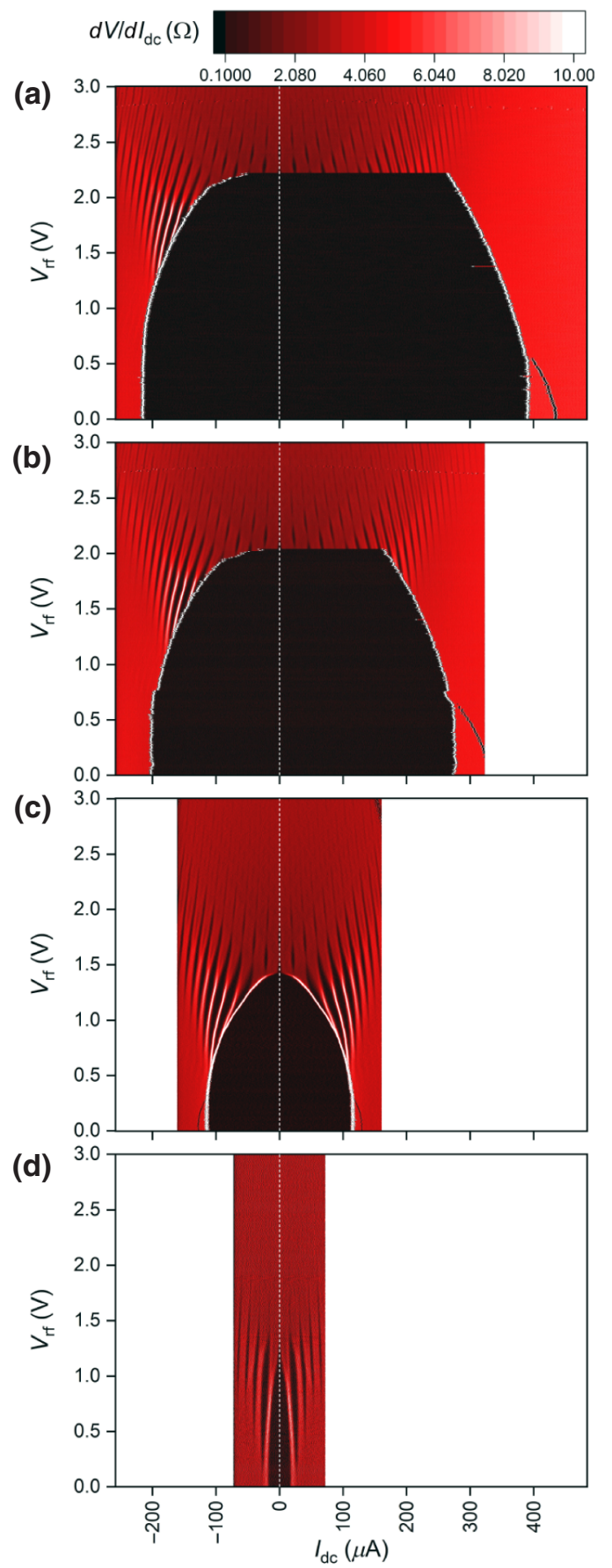

FIG. 16. Differential-resistance color maps taken at (a) $T=$ $6.1 \mathrm{~K}$, (b) $T=6.35 \mathrm{~K}$, (c) $T=6.9 \mathrm{~K}$, and (d) $7.2 \mathrm{~K}$. As in the main text, the dc current sweep is from left to right, that is, the dc current is swept from a finite negative value to a finite positive value. The differential-resistance maps shown in (a) and (b) are taken at temperatures at which the IVCs are hysteretic at zero applied rf current. As the applied rf current is increased, Shapiro steps appear on the dissipative branch of the IVC. As the rf current is increased further, the zeroth Shapiro step is suppressed and the IVC becomes nonhysteretic. The maps shown in (c) and (d) are taken at temperatures at which the IVCs are nonhysteretic at zero applied rf current. Shapiro steps are observed when the rf current is increased. also observed that the amplitude of the applied rf voltage required to fully suppress the zeroth Shapiro step is reduced as the temperature is increased. Note that this device is measured on a different probe from the one used in the main text. Consequently, the $V_{\text {rf }}$ scale is consistent throughout Fig. 16, although it cannot be quantitatively compared to $I_{\mathrm{rf}}$ in Fig. 3 of the main text.

[1] K. K. Likharev, Superconducting weak links, Rev. Mod. Phys. 51, 101 (1979).

[2] R. W. Simmonds, K. M. Lang, D. A. Hite, S. Nam, D. P. Pappas, and J. M. Martinis, Decoherence in Josephson Phase Qubits from Junction Resonators, Phys. Rev. Lett. 93, 077003 (2004).

[3] C. Granata and A. Vettoliere, Nano superconducting quantum interference device: A powerful tool for nanoscale investigations, Phys. Rep. 614, 1 (2016).

[4] L. Hao, C. Aßmann, J. C. Gallop, D. Cox, F. Ruede, O. Kazakova, P. Josephs-Franks, D. Drung, and Th. Schurig, Detection of single magnetic nanobead with a nanosuperconducting quantum interference device, Appl. Phys. Lett. 98, 092504 (2011).

[5] D. Vasyukov, Y. Anahory, L. Embon, D. Halbertal, J. Cuppens, L. Neeman, A. Finkler, Y. Segev, Y. Myasoedov, M. L. Rappaport, M. E. Huber, and E. Zeldov, A scanning superconducting quantum interference device with single electron spin sensitivity, Nat. Nanotechnol. 8, 639 (2013).

[6] A. Finkler, D. Vasyukov, Y. Segev, L. Ne'eman, E. O. Lachman, M. L. Rappaport, Y. Myasoedov, E. Zeldov, and M. E. Huber, Scanning superconducting quantum interference device on a tip for magnetic imaging of nanoscale phenomena, Rev. Sci. Instrum. 83, 073702 (2012).

[7] L. Embon, Y. Anahory, A. Suhov, D. Halbertal, J. Cuppens, A. Yakovenko, A. Uri, Y. Myasoedov, M. L. Rappaport, M. E. Huber, A. Gurevich, and E. Zeldov, Probing dynamics and pinning of single vortices in superconductors at nanometer scales, Sci. Rep. 5, 7598 (2015).

[8] L. Lolli, T. Li, C. Portesi, E. Taralli, N. Acharya, K. Chen, M. Rajteri, D. Cox, E. Monticone, J. Gallop, and L. Hao, Micro-SQUIDs based on $\mathrm{MgB}_{2}$ nano-bridges for NEMS readout, Supercond. Sci. Technol. 29, 104008 (2016).

[9] T. Patel, B. Li, T. Li, R. Wang, J. C. Gallop, D. C. Cox, J. Chen, E. J. Romans, and L. Hao, Toward the use of nanoSQUIDs to measure the displacement of an NEMS resonator, IEEE Trans. Appl. Supercond. 27, 1602005 (2017).

[10] K. Likharev and V. Semenov, RSFQ logic/memory family: A new Josephson-junction technology for sub-terahertzclock-frequency digital systems, IEEE Trans. Appl. Supercond. 1, 3 (1991).

[11] J. M. Williams, D. Henderson, J. Pickering, R. Behr, F. Müller, and P. Scheibenreiter, Quantum-referenced voltage waveform synthesiser, Sci. Measure. Technol. IET 5, 163 (2011).

[12] B. Jeanneret and S. P. Benz, Application of the Josephson effect in electrical metrology, Eur. Phys. J. Spec. Top. 172, 181 (2009).

[13] E. A. Tholén, A. Ergül, K. Stannigel, C. Hutter, and D. B. Haviland, Parametric amplification with weak-link 
nonlinearity in superconducting microresonators, Phys. Scr. 2009, 014019 (2009).

[14] E. M. Levenson-Falk, R. Vijay, and I. Siddiqi, Nonlinear microwave response of aluminum weak-link Josephson oscillators, Appl. Phys. Lett. 98, 123115 (2011).

[15] E. A. Tholén, A. Ergül, D. Schaeffer, and D. B. Haviland, Gain, noise and intermodulation in a nonlinear superconducting resonator, EPJ Quantum Technol. 1, 5 (2014).

[16] R. Vijay, J. D. Sau, M. L. Cohen, and I. Siddiqi, Optimizing Anharmonicity in Nanoscale Weak Link Josephson Junction Oscillators, Phys. Rev. Lett. 103, 087003 (2009).

[17] E. M. Levenson-Falk, F. Kos, R. Vijay, L. Glazman, and I. Siddiqi, Single-Quasiparticle Trapping in Aluminum Nanobridge Josephson Junctions, Phys. Rev. Lett. 112, 047002 (2014).

[18] M. Tinkham, Introduction to Superconductivity, Dover Books on Physics Series (Dover Publications, Incorporated, 2012).

[19] W. J. Skocpol, M. R. Beasley, and M. Tinkham, Selfheating hotspots in superconducting thin-film microbridges, J. Appl. Phys. 45, 4054 (1974).

[20] H. Courtois, M. Meschke, J. T. Peltonen, and J. P. Pekola, Origin of Hysteresis in a Proximity Josephson Junction, Phys. Rev. Lett. 101, 067002 (2008).

[21] V. M. Krasnov, T. Golod, T. Bauch, and P. Delsing, Anticorrelation between temperature and fluctuations of the switching current in moderately damped Josephson junctions, Phys. Rev. B 76, 224517 (2007).

[22] D. Hazra, L. M. A. Pascal, H. Courtois, and A. K. Gupta, Hysteresis in superconducting short weak links and $\mu$ SQUIDs, Phys. Rev. B 82, 184530 (2010).

[23] D. Hazra, J. R. Kirtley, and K. Hasselbach, Retrapping Current in Bridge-Type Nano-SQUIDs, Phys. Rev. Appl. 4, 024021 (2015).

[24] A. Blois, S. Rozhko, L. Hao, J. C. Gallop, and E. J. Romans, Heat propagation models for superconducting nanobridges at millikelvin temperatures, Supercond. Sci. Technol. 30, 014003 (2017).

[25] N. Kumar, T. Fournier, H. Courtois, C. B. Winkelmann, and A. K. Gupta, Reversibility of Superconducting Nb Weak Links Driven by the Proximity Effect in a Quantum Interference Device, Phys. Rev. Lett. 114, 157003 (2015).

[26] N. Kumar, C. B. Winkelmann, S. Biswas, H. Courtois, and A. K. Gupta, Controlling hysteresis in superconducting constrictions with a resistive shunt, Supercond. Sci. Technol. 28, 072003 (2015).

[27] A. Blois, S. Rozhko, L. Hao, J. C. Gallop, and E. J. Romans, Proximity effect bilayer nano superconducting quantum interference devices for millikelvin magnetometry, J. Appl. Phys. 114, 233907 (2013).

[28] S. Biswas, C. B. Winkelmann, H. Courtois, T. Dauxois, H. Biswas, and A. K. Gupta, Elimination of thermal bistability in superconducting weak links by an inductive shunt, Phys. Rev. B 101, 024501 (2020).

[29] S. Biswas, C. B. Winkelmann, H. Courtois, and A. K. Gupta, Josephson coupling in the dissipative state of a thermally hysteretic $\mu$-SQUID, Phys. Rev. B 98, 174514 (2018).

[30] S. Shapiro, Josephson Currents in Superconducting Tunneling: The Effect of Microwaves and Other Observations, Phys. Rev. Lett. 11, 80 (1963).

[31] C. D. Shelly, P. See, J. Ireland, E. J. Romans, and J. M. Williams, Weak link nanobridges as single flux quantum elements, Supercond. Sci. Technol. 30, 095013 (2017).

[32] R. C. Dinsmore III, M.-H. Bae, and A. Bezryadin, Fractional order Shapiro steps in superconducting nanowires, Appl. Phys. Lett. 93, 192505 (2008).

[33] M.-H. Bae, R. C. Dinsmore III, M. Sahu, and A. Bezryadin, Stochastic and deterministic phase slippage in quasione-dimensional superconducting nanowires exposed to microwaves, New J. Phys. 14, 043014 (2012).

[34] J. M. Williams, D. R. Smith, D. Georgakopoulos, P. D. Patel, and J. R. Pickering, Design and metrological applications of a low noise, high electrical isolation measurement unit, IET Sci. Measure. Technol. 3, 165 (2009).

[35] A. De Cecco, K. Le Calvez, B. Sacépé, C. B. Winkelmann, and $\mathrm{H}$. Courtois, Interplay between electron overheating and ac Josephson effect, Phys. Rev. B 93, 180505(R) (2016).

[36] P. Russer, Influence of microwave radiation on currentvoltage characteristic of superconducting weak links, J. Appl. Phys. 43, 2008 (1972).

[37] K. Arutyunov, D. Golubev, and A. Zaikin, Superconductivity in one dimension, Phys. Rep. 464, 1 (2008).

[38] A. Rogachev and A. Bezryadin, Superconducting properties of polycrystalline $\mathrm{Nb}$ nanowires templated by carbon nanotubes, Appl. Phys. Lett. 83, 512 (2003).

[39] C. Kittel, Introduction to Solid State Physics (Wiley, New York, 1986).

[40] J. Wiedenmann, E. Bocquillon, R. S. Deacon, S. Hartinger, O. Herrmann, T. M. Klapwijk, L. Maier, C. Ames, C. Brüne, C. Gould, A. Oiwa, K. Ishibashi, S. Tarucha, H. Buhmann, and L. W. Molenkamp, $4 \pi$-periodic Josephson supercurrent in HgTe-based topological Josephson junctions, Nat. Commun. 7, 10303 (2016).

[41] A. Valizadeh, M. R. Kolahchi, and J. P. Straley, On the origin of fractional Shapiro steps in systems of Josephson junctions with few degrees of freedom, J. Nonlinear Math. Phys. 15, 407 (2008).

[42] A. A. Golubov, M. Y. Kupriyanov, and E. Il'ichev, The current-phase relation in Josephson junctions, Rev. Mod. Phys. 76, 411 (2004).

[43] G. J. Podd, G. D. Hutchinson, D. A. Williams, and D. G. Hasko, Micro-SQUIDs with controllable asymmetry via hot-phonon controlled junctions, Phys. Rev. B 75, 134501 (2007). 